Albrecht v. Graefes Arch. klin. exp. Ophthal. 197, 25-30 (1975)

(C) by Springer-Verlag 1975

\title{
Drusen of the Iris: in Advanced Malignant Choroidal Melanoma*
}

\author{
J. Reimer Wolter and Ronald D. Cox \\ The Departments of Ophthalmology and Pathology of the University of Michigan Medical \\ Center, Ann Arbor, Michigan 48104
}

Received October 20, 1974

Summary. Drusen were found in the iris between the layers of the pigment epithelium and the dilator muscle in an eye of a 52 year old patient that contained a large malignant choroidal melanoma. The significance of this unusual observation in comparison to the common drusen of Bruch's membrane of the choroid is discussed.

Zusammenfassung. In einem Auge einer 52jährigen Patientin, das wegen eines Melanoms der Aderhaut enukleiert werden mußte, fanden sieh Drusen der Regenbogenhaut, die zwischen dem Pigmentepithel und dem Dilatator-Muskel lagen. Die Bedeutung dieser Drusen im Vergleich zu den Drusen der Aderhaut wird erörtert.

Drusen in the region of the choroid are well known to all ophthalmologists as button-like hyaline deposits on Bruch's membrane which may be seen ophthalmoscopically as round yellowish spots deep to the retina and often surrounded by borders of increased pigmentation. Drusen in the neuroectodermal layers of the iris, in contrast, appear to be a rarety. I have never seen any before and I have been unable to find reports about drusen of the iris in the literature.

To report the occurrence of numerous drusen between the layers of the pigment epithelium and the dilatator muscle of the iris in an eye with a large malignant melanoma is the purpose of the present paper.

\section{Case Report}

This 52 year-old white female patient had noticed slowly progressive loss of vision in her right eye for more than two years. For several months the eye had been totally blind. Pain developed in the eye recently and this brought the patient to see an ophthalmologist. Clinical examination revealed secondary glaucoma, early rubeosis iridis and retinal detachment that was solid in some parts and exudative in others. In the eareas of solid detachment a large dark tumor was recognized under the retina. A clinical diagnosis of advanced malignant choroidal melanoma causing secondary glaucoma was made and enucleation was advised. The left eye was normal. It was of special interest that occasional small drusen were recognized ophthalmologically in the retinal periphery of this normal left eye.

The right eye was enucleated without difficulties in general anaesthesia on 6-19-74. Inspection of the removed right eye did not reveal any evidence of extraocular tumor extension.

Pathological study showed the removed eye to contain a round dark choroidal tumor measuring $13 \times 9 \times 10 \mathrm{~mm}$ that was protruding from the choroid into the retroretinal space. The retina was detached. Microscopic study of sections stained with Hematoxylin Eosin and PAS Schiff stain gave the following results.

\footnotetext{
* Supported by the Research to Prevent Blindness, Ine., New York, N.Y.
} 

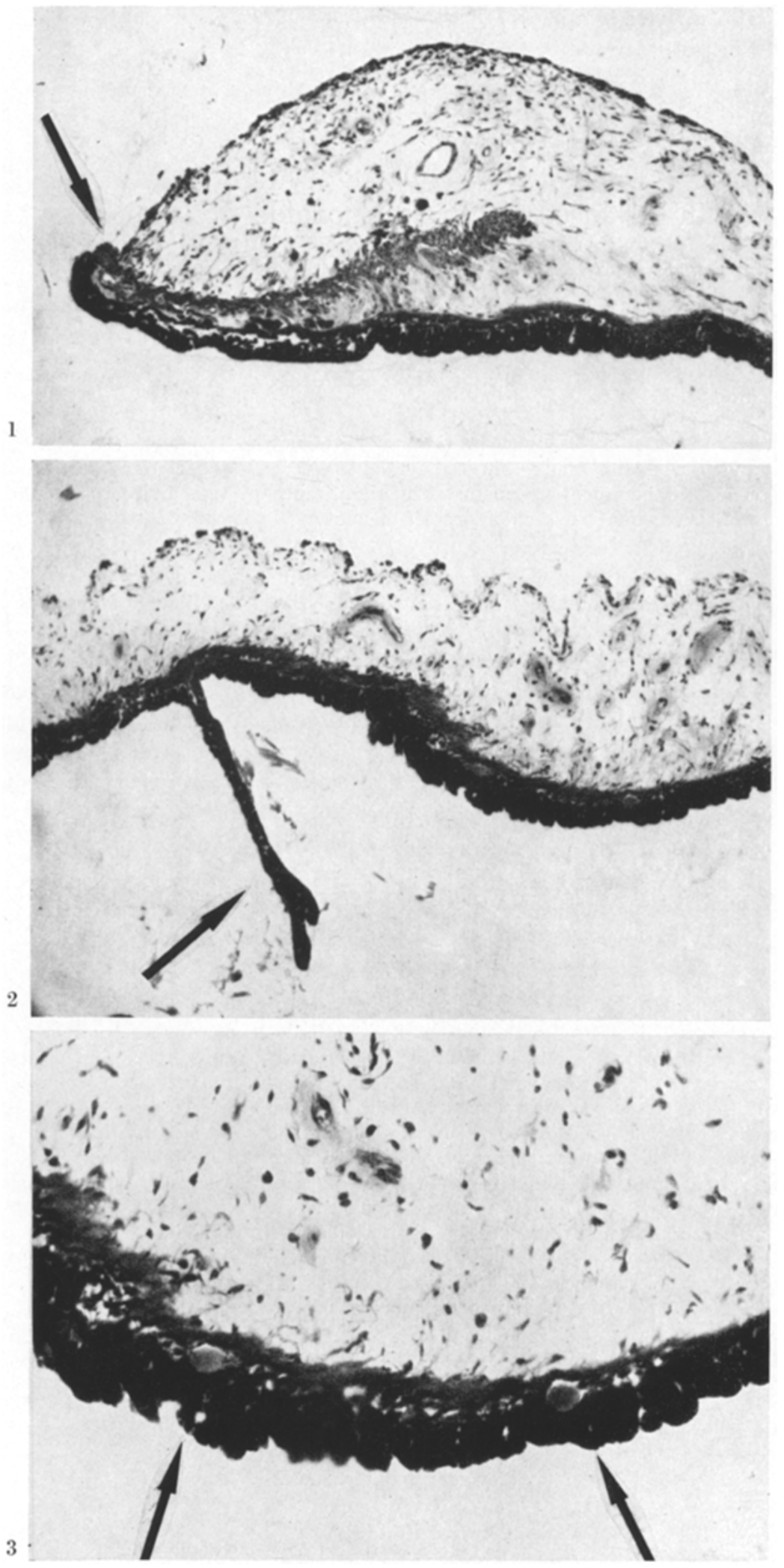


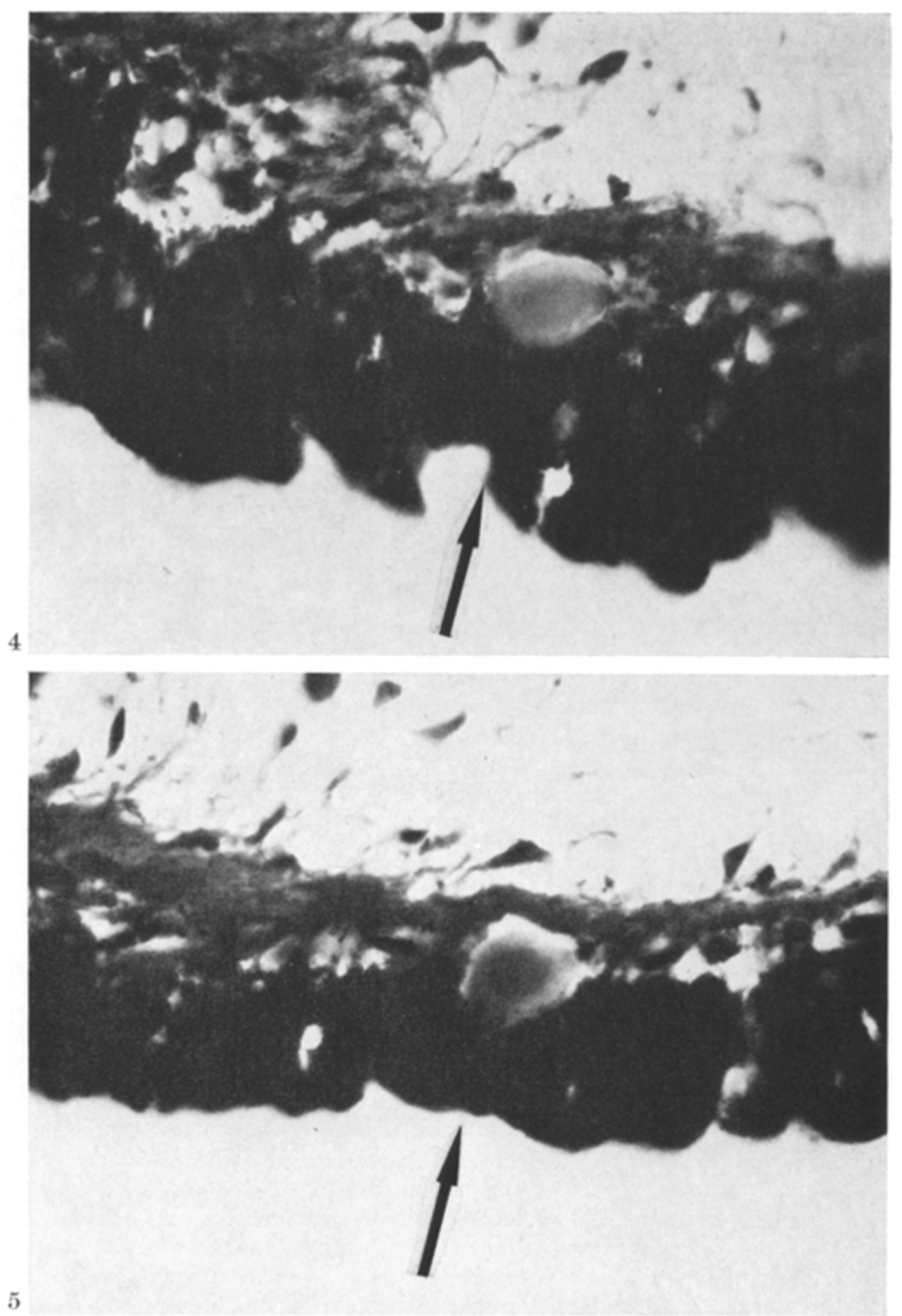

Fig. 1. Low power of cross section of the iris showing slight extropion uveae (arrow). -paraffin section, $\mathrm{H}$ and $\mathrm{E}$ stain, photomicrograph $\times 100$

Fig. 2. Displaced ciliary process on the peripheral iris (arrow). -paraffin section, $\mathrm{H}$ and $\mathrm{E}$ stain, photomicrograph $\times 100$

Fig. 3. Two drusen (arrows) located between pigment epithelium and dilatator muscle of the iris. -paraffin section, $H$ and $\mathbf{E}$ stain, Photomicrograph $\times 250$

Fig. 4. Druse of the iris in the present case (arrow) seen at higher power. -paraffin section, $\mathrm{H}$ and $\mathrm{E}$ stain, photomicrograph $\times 600$

Fig. 5. Another druse in the iris of the present case (arrow) with distinctly layered strueture. -paraffin section, $\mathrm{H}$ and $\mathrm{E}$ stain, photomicrograph $\times 600$ 


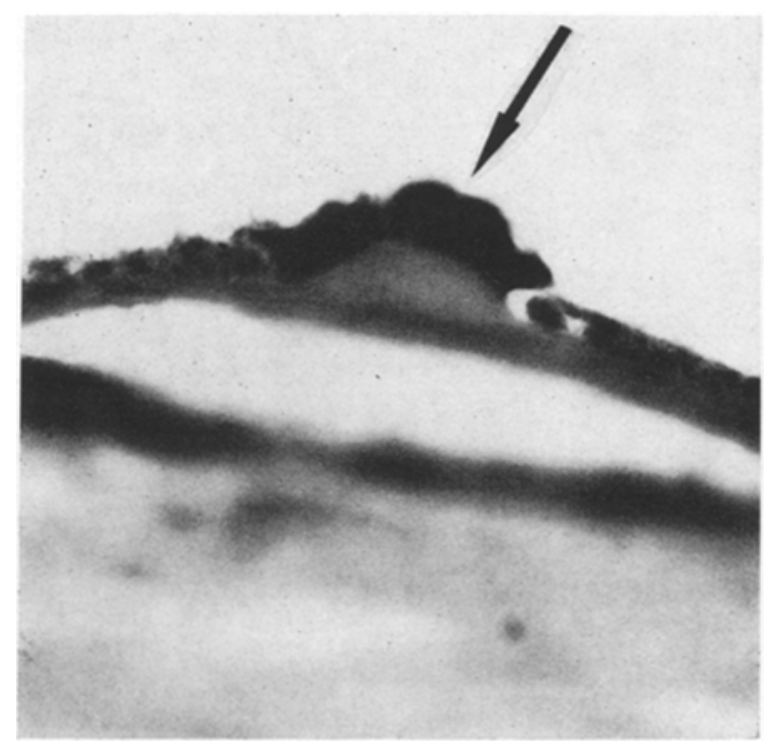

Fig. 6. Druse on Bruch's membrane of the choroid (arrow) in the present case. -paraffin section, $\mathrm{H}$ and $\mathrm{E}$ stain, photomicrograph $\times 600$

The corneal epithelium exhibited an erosion and a few areas with bullous changes. Bowman's membrane was continuous. The corneal stroma appeared to be slightly edematous. Descemet's membrane was continuous and the corneal endothelium was about normal. The iris exhibited some thin-walled blood vessels on its anterior surface and the anterior chamber angle was narrow. Early ectropin uveae was recognized on the pupillary border (Fig. 1). Occasional lymphocytes and plasma cells were found in the iris stroma. A displaced eiliary process was seen on the peripheral iris (Fig. 2).

Surprisingly, numerous drusen were observed in the iris in a zone between the pigment epithelium and the myo-epithelial cells of the dilatator muscle (Figs. 3, 4 and 5). These drusen were round bodies of hyaline substance which gave the typical stains with both, the $\mathrm{H}$ and $\mathrm{E}$ and the PAS stains and exhibited the lamellar structure (Fig. 5) that is typically seen in drusen. Both layers, the pigment epithelium and the dilatator muscle were well preserved in the region of the drusen as well as elsewhere in this iris.

The ciliary body, showed some mononuclear infiltration as well as some distortion due to the extensive retinal detachment. The lens exhibited early subcapsular cataractous changes. The vitreous was involved with advanced degeneration. The detached retina showed neuronal atrophy, gliosis and distortion everywhere. It was directly involved with the large choroidal tumor that had broken through Bruch's membrane and was composed of spindle-shaped cells mostly. Some of these cells contained pigment. Most of the cells had a nucleolated nucleus. Serous exudate was found in the retro-retinal space surrounding the tumor. The tumor had at its base in the mid-periphery extended into the inner most layers of the sclera, but it had not grown through the sclera to the outside of the eye. The optic nerve was not directly involved, but it exhibited distinct atrophy.

It was of special interest that the pigment epithelium in the region of the choroid exhibited many small drusen (Fig. 6) which were in size and staining characteristics very similar to the drusen of the iris.

The histopathological diagnosis, thus, was: large malignant choroidal melanoma of a spindle B cell type without direct extraocular extensions. The eye also exhibited bullous keratopathy, early rubeosis iridis, early ectropin uveae, partly exudative and partly solid retinal detachment with secondary atrophy of retina and optic nerve. Small drusen of the 
senile type were observed on the choroid and, surprisingly, very similar drusen were also found in the iris between pigment epithelium and the myo-epithelial cells.

\section{Discussion}

The first extensive histological study of drusen of Bruch's membrane of the choroid was done in 1855 by $H$. Mueller [1]. These drusen are almost constant in people over 60 years of age, common in people over 45 years and not unusual in younger people. The literature contains more than a dozen different theories to explain the development of drusen. The space of this paper does not allow for a discussion of all of them, but three major theories have survived and deserve attention.

Mueller [1] and Alt [2] suggested drusen were a secretion of an intact pigment epithelium. Donders [3] proposed the transformation theory by suggesting they were the result of the direct conversion of degenerating pigment epithelium into drusen material. A vascular theory of drusen formation has been advanced by Friedman, Smith, and Kuwabara [4] who noted pink staining bodies around collecting venules of vortex veins. They believed these bodies to be exudative material which result from vascular decompensation in the aged and which then migrate through porous spaces in the lamina vitrea to be deposited beneath the pigment epithelium.

Relatively recent work by Hogan $[5,6]$ in electron microscopy and further such studies including the histochemical characterization of drusen by Farkas, Sylvester, Archer and Altona [7, 8] have further eluciated the nature of drusen and the probable mechanism of their formation. Hogan $[5,6]$ has observed that drusen material accumulates in the retinal pigment epithelium cells and in the region of Bruch's membrane. Farkas, Sylvester and Archer [7] have shown that the retinal pigment epithelial cells degenerated into an amorphous material which fills the inner collagenous zone of Bruch's membrane forming drusen. They have demonstrated that a large number of lysosomes are present in the degenerating retinal pigment epithelial cells and drusen material. This, they suggest, supports the theory that drusen formation is due to uncontrolled lysosomal cytolysis of retinal pigment epithelium. It is also noted by Farkas, Sylvester and Archer [7] that the choriocapillaries layer is free from drusen material and that the elastic lamina of Bruch's membrane prevents passage of this material. Thus the site of drusen formation could not be choriocapillaries as suggested by Friedman, Smith and Kuwabara [4].

Histochemical studies by Farkas, Sylvester, Archer and Altona [8] have shown the composition of drusen of Bruch's membrane to consist of the mucopolysaccharide, sialic acid, and a lipid found to be cerebroside. They suggest that drusen are formed by abnormal lysosomal activity causing the autophagic destruction of retinal pigment epithelium. Mitochondria contain sialic acid. Retinal pigment epithelium contains lipofuscin granules which could be a source of cerebrosides. Thus the presence of the two major constituents of drusen is explained.

The above-mentioned findings are of special interest if one considers their possible application to the present case of typical drusen between the pigment

3 Albrecht v. Graefes Arch. klin. exp. Ophthal. 
epithelium and dilator muscle of the iris. This may represent uncontrolled lysosomal cytolysis of the iris pigment epithelium. This iris drusen in the present case of ocular melanoma may, of course, have to be classified as so-called secondary drusen [9].

The occurrence of drusen in the iris appears to be extremely rare, however, and thus it would not be wise to make more speculations and conclusions concerning this unusual finding in a single eye with rubeosis as well as a large intraocular tumor. All emphasis should be one the most interesting observation of these drusen under the conditions of the present case.

\section{References}

1. Mueller, H.: Anatomische Beiträge zur Ophthalmologie. Arch. Ophthal. 2, 1 (pt 2) (1855)

2. Alt, A.: Beiträge zur pathologischen Anatomie des menschlichen Auges. Arch. Ophthal. Otolaryng. 6, 304 (1877)

3. Donders, F.C.: Beiträge zur pathologischen Anatomie des Auges. Arch. Ophthal. 1, 106 (Pt. 2) (1855)

4. Friedman, E., Smith, T., Kuwabara, T.: Senile choroidal vascular patterns and drusen. Arch. Ophthal. 69, $114(1963)$

5. Hogan, M. J.: Bruch's membrane and disease of the macula. Role of elastic tissue and collagen. Trans. ophthal. Soc. U.K. 87, 113 (1967)

6. Hogan, M. J., Alvarado, J. A., Weddell, J. E.: Histology of the human eye, p. 344-363. Philadelphia: W. B. Saunders 1971

7. Farkas, T. G., Sylvester, V., Archer, D.: The ultrastructure of drusen. Amer. J. Ophthal. 71, 1196 (1971)

8. Farkas, T. G., Sylvester, V., Archer, D., Altona, M.: The histochemistry of drusen. Amer. J. Ophthal. 71, 1206 (1971)

9. Wolter, J. R., Falls, H. F.: Bilateral confluent drusen. Arch. Ophthal. 68, 219 (1962)

J. Reimer Wolter, M. D.

Departments of Ophthalmology

and Pathology

University of Michigan

Medical Center

Ann Arbor, Michigan 48104, USA 